Journal of Engineering and Applied Sciences 14 (10): 3322-3325, 2019

ISSN: 1816-949X

(C) Medwell Journals, 2019

\title{
Activation Energy of Poly (Ethylene Oxide) Dispersed with Carbon Black Nanoparticles
}

\author{
Husam Miqdad \\ Department of Basic Science, Applied Science Private University, Amman, Jordan
}

\begin{abstract}
Electrical properties of hybrid polymer thin films, made of Poly Ethylene Oxide PEO doped with conductive Carbon Black (CB) nanoparticles $(0.1 \mathrm{wt} \%$ ) have been investigated. The observed physical constants of the casted thin films like activation energy were determined. The prepared films by casting method have been electrically. The present study has studying the variation of activation energy with frequency in the range $(200-1000 \mathrm{kHz})$ and temperature in the range $\left(30-55^{\circ} \mathrm{C}\right)$ for $(\mathrm{PEO})$ with doped $0.1 \mathrm{wt} . \%$ carbon black as compared to that case of the un doped ( $\mathrm{PEO}$ ) film. The observed values of the activation Energy $\left(\mathrm{E}_{\mathrm{a}}\right)$ showed frequency and temperature. It was found that the activation energy of the prepared thin films decrease with doped $(0.1$ wt. \%) Carbon Black (CB) complexes and that it decrease with frequency and temperature according to polarization processes.
\end{abstract}

Key words: $\mathrm{PEO}, \mathrm{CB}$, activation energy, frequency, temperature, polarization

\section{INTRODUCTION}

Most polymeric materials are poor conductors of electricity because of the unavailability of large numbers of free electrons to participate in the conduction process. So, a great attention has been focused on enhancing their electrical conduction and improving their properties (Callister and William, 2007). Polymers exhibit a variety of electrical properties reflecting their structure and molecular motion. Electrical properties can be tailored to a specific requirement by the addition of suitable dopant materials. Conductive Polymer Composites (CPC) have been given great interest in many industrial applications owing to their desirable properties which made them favorable compared to other commercial materials. One important aspect of these characteristics is the transition from insulating to conductive behavior, through addition of some conducting substances as metallic particles carbon or steel fibers, aluminum flakes and carbon black.

Poly Ethylene Oxide (PEO) is a nonionic homopolymer of ethylene oxide, represented by the formula $\left(-\mathrm{CH}_{2} \mathrm{CH}_{2} \mathrm{O}-\right)^{\mathrm{n}}$. In which $\mathrm{n}$ represents the average number of oxy-ethylene groups and its structure is (Fig. 1). Poly (Ethylene Oxide) (PEO) is a synthetic polyether that is readily available in arrange of Molecular

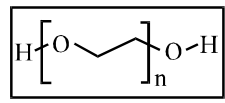

Fig. 1: PEO structure weights (MW). Material with $\mathrm{MW}<100,000 \mathrm{~g} / \mathrm{mol}$ are usually called Poly (Ethylene Glycol) PEG while higher molecular weight polymer are classified as PEO. These polymers are soluble in water as well as in many organic solvents (e.g., methylene chloride, ethanol, toluene, acetone and chloroform). PEOs are white solids with melting point proportional to their molecular weights. The interesting properties of $\mathrm{PEO}$ are its relatively low melting point $65^{\circ} \mathrm{C}$, good structural integrity, low glass transition temperatures $-65^{\circ} \mathrm{C}$ which permits ion transport at ambient temperature, low toxicity. On other hand, PEO is the most popular polymer used, due to its high solvating power with metal ions, good process ability and outstanding mechanical properties, it has a lot of trade names (Alathen, Fortiflex, Zendel) (www.Polymerdata.Com). Poly (ethylene oxide) is a familiar example of the thermoplastic polymers and these can be further sub-divided into amorphous and crystalline polymers structures (Table 1).

Carbon black is a conductive material and is largely composed of carbon atoms or aggregates of carbon atoms, although, considerable quantities of hydrogen and oxygen can also be present. Carbon black are approximately spherical but the others have complex three-dimensional shapes which can be thought of as the partial fusing together of a number of nanometer-sized spherical primary particles (Rothon, 1995). Carbon black

\begin{tabular}{ll} 
Table 1: Physical properties of Poly Ethylene Oxide PEO & \\
\hline$*$ Appearance & White powder \\
\hline *Melting point (molecular weight $>(20000))$ & $65^{\circ} \mathrm{C}$ \\
**Refractive index & 1.456 \\
**Dielectric constant & 4.5 \\
Glass transition Temperatures $\left(\mathrm{T}_{q}\right)$ & $-65^{\circ} \mathrm{C}$ \\
\hline$*$ ** Significant values &
\end{tabular}


has been added to materials to reduce their electrical resistance. It is supposed that this property is a consequence of the $\mathrm{CB}$ particles structure linking each other which compose conductive channels or in order to the "tunnel effect" of electrons jumping between dispersed particles of carbon black. The formation of a continuous chain of the $\mathrm{CB}$ particles with electronic movement occurring along this continuous chain is due to inter-particular contact (conduction path theory). In addition to the above, conduction can occur due to the ability of electrons to "jump" across gaps existing between carbon black particles within the polymer matrix (tunnel effect theory) (Crossman, 2004).

In this study, the activation energy mechanism in doped poly ethylene oxide with $\mathrm{CB}$ was investigated and the results were reported and discussed. In continuation of these studies, the activation energy mechanism in PEO doped with $\mathrm{CB}$ films was verified as function of frequency and is discussed in this paper by acceptors and holes and electrons produced by valence-band disruption.

\section{MATERIALS AND METHODS}

Experimental work: In this study the material examined is (poly ethylene oxide) thin films doped with carbon black as dopant and neat PEO sample for contrast the noticed consequences. The objective of the research is to investigate the activation energy of casted Poly ethylene oxide thin films doped with conducting carbon black as compared to that case of the un doped (PEO) film.

Preparation of thin films: $\mathrm{PEO}$ and carbon black powders were blended together in methanol as a convenient solvent. Then for 2 days the mixture was mixed by using a rotary magnet to get a homogeneous mixture. On to a glass mould the mixture was directly casted to delicate films. At room temperature by waiting for 2 days the methanol was permitted to evaporate perfectly. All samples were dried in the oven at temperature $40^{\circ} \mathrm{C}$ for 2 days.

Measurements of electrical properties: Hewlett Packard (HP) 4192A impedance analyzer was used to measure impedance and phase angle values by varying the applied frequency (ranges from $5 \mathrm{~Hz}$ up to $13 \mathrm{MHz}$ ). The specimen was placed firmly between two copper electrodes in a sample holder shown in Fig. 2. These electrodes are connected through cables to the impedance analyzer. Impedance measurements were performed in a frequency range from about $(200-1000 \mathrm{kHz})$ over a temperature range $\left(30-55^{\circ} \mathrm{C}\right)$ with steps of $5^{\circ} \mathrm{C}$. Since, the melting Temperature $\left(\mathrm{T}_{\mathrm{m}}\right)$ for $\mathrm{PEO}$ is about $60^{\circ} \mathrm{C}$ no higher temperature measurements were conducted. Temperature readings were taken in a steady state condition.

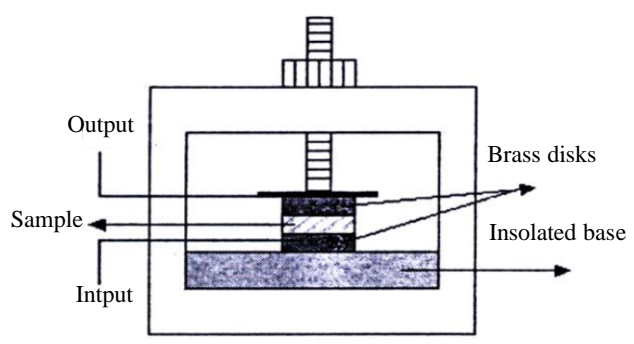

Fig. 2: The sample holder diagram Impedance analyze

Dielectric materials are a special class of substances that, under almost all conditions are insulators. They have the interesting and useful property that their electrons, ions or molecules may be polarized under the influence of an external electric field. When such materials are placed between charged plates as in capacitors, they increase the total capacity of these devices. This application constitutes one of the important applications of these materials (Pollock, 1993).

Connecting a Capacitor (C) to a Resistor (R) in parallel, the impedance $(Z)$, the real component of the impedance $\left(Z^{\prime}\right)$ and the imaginary component of the impedance (Z") of the circuit are:

$$
\begin{aligned}
& Z=\frac{R(1-i \omega C R)}{1+(\omega C R)^{2}} \\
& Z^{\prime}=\frac{R}{1+(\omega C R)^{2}} \\
& Z^{\prime \prime}=\frac{\omega C R^{2}}{1+(\omega C R)^{2}}
\end{aligned}
$$

The dielectric constant $\left(\varepsilon^{\prime}\right)$ which is related to the stored energy within the medium and the dielectric loss (E") which is related to the loss of energy within the medium in form of heat generated by an electric field are determined from these relations (Elimat, 2006).

$$
\begin{aligned}
& \varepsilon^{\prime}=\frac{Z^{\prime \prime}}{2 \pi \mathrm{fC}_{0} \mathrm{Z}^{2}} \\
& \varepsilon^{\prime \prime}=\frac{\mathrm{Z}^{\prime}}{2 \pi f \mathrm{C}_{0} \mathrm{Z}^{2}}
\end{aligned}
$$

Where:

$\mathrm{C}_{0}=$ The Capacitance without the thin film

$\mathrm{f}=$ The frequency $(\mathrm{AC})$ of electric field

The AC conductivity $\left(\sigma_{A C}\right)$ of the thin film is given by the relation: 


$$
\sigma_{\mathrm{AC}}=2 \pi f \varepsilon_{0} \varepsilon^{\prime \prime}
$$

Using Arrhenius equation, the activation Energy $\left(\mathrm{E}_{\mathrm{a}}\right)$ of the conduction process is determined by Hummel (1993):

$$
\sigma=\sigma_{0} \exp \left[-\mathrm{E}_{\mathrm{a}} / \mathrm{k}_{\mathrm{B}} \mathrm{T}\right]
$$

Where:

$\mathrm{k}_{\mathrm{B}}=$ The Boltzmann constant

$\mathrm{T}=$ The Temperature in Kelvin

$\sigma_{0}=$ The material conductivity constant

$\sigma=$ The electrical conductivity

\section{RESULTS AND DISCUSSION}

A dopant carbon black appended to the matrix of polyethylene oxide to compose solid electrolyte thin films is being searched to evaluate the role of the doping in the process of the activation energy the electric field is affected. The objective of studying activation energy in polymers is to realize the type and nature of the charge transmission in conducting materials (Agrawal and Pandey, 2008).

Activation energy determination: The minimum amount of thermal energy that is required to activate ions, atoms and molecules to conduction in which they can undergo physical transport is known as activation Energy $\left(\mathrm{E}_{\mathrm{a}}\right)$. The values of activation energy were determined from the slopes of the straight lines obtained by drawing the natural logarithm of the conductivity $\left(\operatorname{Ln} \sigma_{\mathrm{AC}}\right.$ ) versus $(1000 / \mathrm{T})$ for all thin films at the frequencies $(200,400,600$, 800 and $1000 \mathrm{kHz}$ ) as shown in Fig. 3 and 4.

By using Arrhenius equation the activation energy values were calculated and summarized reported in Table 2 and 3 which display the relation between activation energy and frequency for thermally activated conduction processes (ionic and electronic). It was observed that with increasing the frequency, the activation energy decreases for all tested composites. The decrease in activation energy reflects electronic mobility, higher ionic and increasing in electrical conduction in the samples membranes (Ahmad et al., 1991).

Figure 5 displays that with doped $0.1 \mathrm{wt} . \%$ carbon black the activation energy value decreases in a thermally activated process, the thin films will have high conductivity as seen in Fig. 7. This noticed reduction in activation energy value means that the polyethylene oxide/CB thin films energy will be narrower, referring to production of localized energy states by heating which increases the ability of the electron to tunnel or jump
Table 2: The activation energy values for neat PEO

\begin{tabular}{lc}
\hline Frequency $(\mathrm{kHz})$ & Activation energy $\times 10^{2}(\mathrm{eV})$ \\
\hline 200 & 32.4 \\
400 & 31.6 \\
600 & 30.7 \\
800 & 25.8 \\
1000 & 23.2 \\
\hline
\end{tabular}

Table 3: The activation energy values for $\mathrm{PEO}+0.1 \% \mathrm{CB}$

\begin{tabular}{lc}
\hline Frequency $(\mathrm{kHz})$ & Activation energy $\times 10^{-2}(\mathrm{eV})$ \\
\hline 200 & 30.9 \\
400 & 29.1 \\
600 & 28.3 \\
800 & 24.7 \\
1000 & 23.6 \\
\hline
\end{tabular}

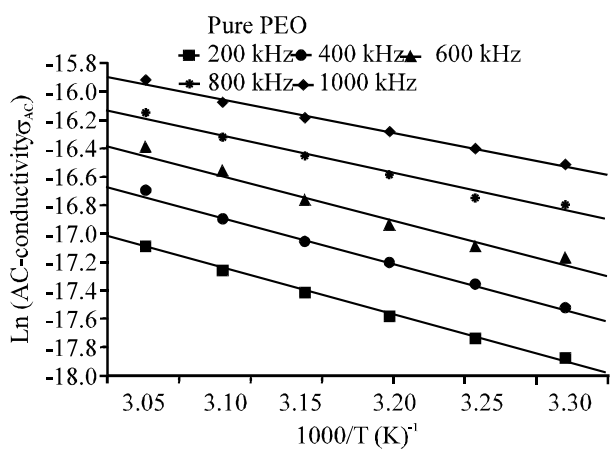

Fig. 3: Ln (AC-conductivity) versus $(1000 / \mathrm{T})$ for neat $\mathrm{PEO}$

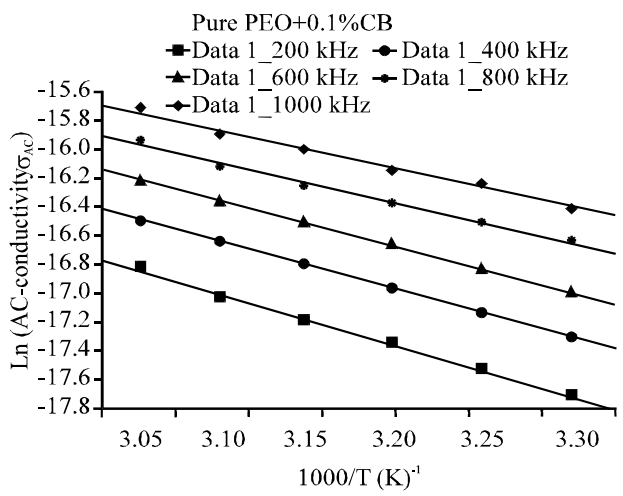

Fig. 4: $\operatorname{Ln}($ AC-conductivity) versus (1000/T) for $\mathrm{PEO} / \mathrm{CB}$

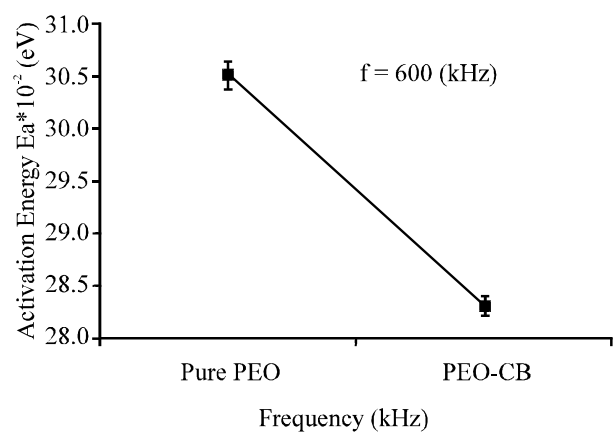

Fig. 5: Variation of $\mathrm{E}_{\mathrm{a}}$ values at $600 \mathrm{kHz}$ 


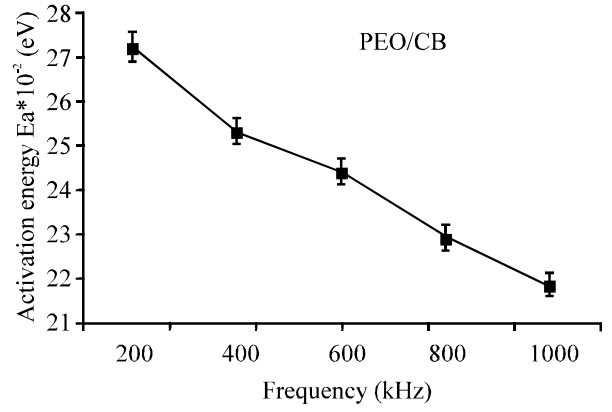

Fig. 6: Variation of $\left(\mathrm{E}_{\mathrm{a}}\right)$ values with frequency for $\mathrm{PEO} / \mathrm{CB}$ composite

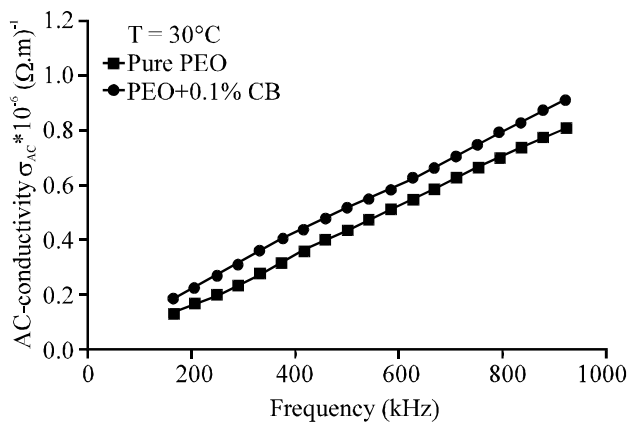

Fig. 7: Dependence of $\mathrm{AC}$ conductivity as a function of frequency

from the valence to conduction energy band and increases ion mobility. These events increase the amorphous region in the polymer $\mathrm{PEO}$ and produce more active segmentals motion which stimulates the electron hopping mechanism and thus, the conductivity becomes higher. In our study, the noticed increase in conductivity and reduction in the $\left(\mathrm{E}_{\mathrm{a}}\right)$ values with increasing temperature or the carbon black content could be controlled by the mobility in the localized positions bridged by the particles of carbon black in the amorphous regions present in the polymer electrolyte. The low $\left(\mathrm{E}_{\mathrm{a}}\right)$ for charges in thecarbon black transition refers to the amorphous nature of polymer which simplifies the rapid electrons movements in the polymer complex (Sharma et al., 2007).

Figure 6 displays that with increasing the frequency the activation Energy $\left(E_{a}\right)$ decreases because at higher field, more charges are moved, causing more participation of activated charges to conduction. It can be observed that $\left(\mathrm{E}_{\mathrm{a}}\right)$ is decreased at higher frequency and with doped 0.1 wt. \% carbon black.

\section{CONCLUSION}

The activation energy of PEO thin films doped with carbon black was studied. By studying the results, we deduced that:
- The activation energy decreases with doped carbon black nanoparticles

- The activation energy is decreased at higher frequency

- The activation energy is decreased with increasing temperature

\section{ACKNOWLEDGEMENT}

The research acknowledges Applied Science Private University Amman, Jordan, for the fully financial support granted of this research article.

\section{REFERENCES}

Agrawal, R.C. and G.P. Pandey, 2008. Solid polymer electrolytes: Materials designing and all-solid-state battery applications: An overview. J. Phys. D. Appl. Phys., 41: 223001-223001.

Ahmad, M.S., M.H. Abdallah, A.M. Zihlif and M.K. Abdelazeez, 1991. Electrical properties of oilshale rocks. J. Mater. Sci., 26: 203-207.

Callister, William, D., 2007. Material Science and Engineering: An Introduction. 4th Edn., John Wiley and Sons, New York, USA.,

Crossman, R.H., 2004. Conductive polymer composites polym. J. Eng. Sci., 25: 507-513.

Drake, N., 1996. Polymeric Materials for Electrostatic Applications. Rapra Technology Ltd, Priorslee, England, UK., Pages: 163.

Elimat, Z.M., 2006. Electrical and thermal conductivity of poly (methyl methacrylate)/carbon black composites. Mutah Li. Buhuth Wad. Dirasat, 21: 169-182.

Hummel, R.E., 1993. Electronic Properties of Materials: An Introduction for Engineers. 2nd Edn., Springer, Berlin, Germany, ISBN: 9783540548393 , Pages: 404.

Pollock, D.D., 1993. Physical Properties of Materials for Engineers. 2nd Edn., CRC Press, USA., ISBN-10: 0849342376, pp: 608 .

Rothon, R.N., 1995. Particulate-Filled Polymer Composites. 1st Edn., Longman Scientific \& Technical, England, UK., ISBN:9780582087828, Pages: 375 .

Sharma, A.K., P.B. Bhargav, V.M. Mohan and V.N. Rao, 2007. Structural, electrical and optical characterization of pure and doped poly (vinyl Alcohol) (PVA) polymer electrolyte films. Intl. J. Polym. Mater., 56: 579-591. 